\title{
RESPIRATORY INFECTION
}

\section{Short term outcome and risk factors for adverse clinical outcomes in adults with severe acute respiratory syndrome (SARS)}

\author{
J W M Chan, C K Ng, Y H Chan, T Y W Mok, W H O, S Lee, S Y Y Chu, W L Law, \\ M P Lee, P C K Li
}

Thorax 2003;58:686-689

See end of article for authors' affiliations

.....................

Correspondence to: Dr J W M Chan, Department of Medicine, Queen Elizabeth Hospital, 30 Gascoigne Road, Kowloon, Hong Kong; johnnychan@excite.com

Final version received 14 May 2003

Accepted for publication

16 June 2003

\begin{abstract}
Background: Severe acute respiratory syndrome (SARS) was diagnosed in Hong Kong in over 1700 patients between March and early June 2003.

Methods: 115 patients diagnosed with SARS were admitted to Queen Elizabeth Hospital, a large regional hospital in Hong Kong, from March 2003, of whom 100 were either discharged or were dead at 31 May. The patients were prospectively studied after admission to assess their short term outcomes and the risk factors associated with adverse outcomes, defined as death or the need for mechanical ventilation

Results: At the time of writing 18 patients had died, with a crude mortality rate of $15.7 \%$ and a 21 day mortality of $10 \%$ (standard error $3 \%$ ). Thirty nine patients $(34 \%$ ) were admitted to the intensive care unit, 30 of whom $(26 \%)$ required mechanical ventilation. Multivariate analysis showed that age above 60 (hazards ratio (HR) $3.5,95 \% \mathrm{Cl} 1.2$ to $10.2 ; \mathrm{p}=0.02$ ), presence of diabetes mellitus or heart disease (HR 9.1, 95\% Cl 2.8 to 29.1; $\mathrm{p}<0.001)$, and the presence of other comorbid conditions (HR $5.2,95 \% \mathrm{Cl} 1.4$ to $19.7 ; \mathrm{p}=0.01$ ) were independently associated with mortality. However, only the presence of diabetes mellitus and/or cardiac disease (HR 7.3,95\% $\mathrm{Cl} 3.1$ to $17.4 ; \mathrm{p}<0.001$ ) was associated with adverse outcomes as a whole.

Conclusion: SARS is a new disease entity that carries significant morbidity and mortality. Specific clinical and laboratory parameters predicting unfavourable outcomes have been identified.
\end{abstract}

S evere acute respiratory syndrome (SARS) caused by a novel coronavirus ${ }^{1-3}$ has been described in Hong Kong since March 2003, and several series of patients have been described in the literature. ${ }^{4-7}$ Between 9 March and 31 May, 115 adults were admitted to our hospital with a diagnosis of clinical SARS. A treatment protocol including a combination of ribavirin and systemic steroids was adopted from the beginning of the epidemic, in accordance with the guidelines of the Hospital Authority of Hong Kong. ${ }^{8}$ Unfortunately, without a definitely reliable treatment regimen (at least in the initial phase), the number of admissions to the intensive care unit (ICU) and the mortality rate have been high.

We describe the clinical features and short term outcomes of these patients, and attempt to identify the initial presenting factors that might account for the adverse clinical outcomes.

\section{METHODS}

All patients admitted to our hospital who fulfilled the WHO criteria for probable SARS were included in the study. ${ }^{9}$ In brief, the definition of SARS is: fever of $38^{\circ} \mathrm{C}$ or higher at presentation or during the previous 2 days; new pulmonary infiltrates on the chest radiograph or high resolution CT scan, with or without chills or rigours; cough or shortness of breath; presence of contact history; and the absence of an alternative diagnosis to explain the clinical presentation.

On admission a standard chart was used to record the demographic and clinical information of the patients including age, sex, exposure history, occupation, symptoms, physical findings, and initial chest radiographic presentation. Laboratory investigations included complete blood picture with differential counts, clotting profiles, biochemical tests (liver and renal function, electrolytes, creatine kinase, and lactate dehydrogenase), microbiological tests to exclude other pathogens as the aetiological agents (bacterial cultures of sputum, blood and urine, serological tests for Mycoplasma, Chlamydia, Legionella, influenza, parainfluenza, respiratory syncytial virus and adenovirus, nasopharyngeal swab or aspirate for rapid antigen testing of influenza, sputum for AFB smear and culture). A reverse transcriptase polymerase chain reaction (RT-PCR) test for SARS virus was performed on diagnosis of clinical SARS for respiratory samples (nasopharyngeal aspirates, throat swabs or tracheal aspirates) as well as a stool sample if diarrhoea was present. Blood samples for coronavirus IgG antibodies (immunofluorescent assay) were also obtained on the day of diagnosis and initiation of SARS treatment, and on days 7, 14, and 21 or upon discharge. Chest radiography was performed in all patients at least once daily throughout the admission and assessed by the same respiratory team. A high resolution CT scan of the thorax was performed for early diagnosis of pneumonic changes in cases were there was a definite history of exposure with persistent fever but normal plain chest radiographs.

All patients were treated with broad spectrum antibiotics including a $\beta$-lactam/ $\beta$-lactamase inhibitor (amoxicillinclavulanate) or a cephalosporin (cefotaxime or ceftriaxone), together with a macrolide (either azithromycin or clarithromycin). Levofloxacin was used in patients where there was a history of $\beta$-lactam allergy. Intravenous ribavirin $400 \mathrm{mg}$ was given every 8 hours for 10-14 days and systemic steroids (starting with intravenous hydrocortisone $200 \mathrm{mg}$ every 6-8 hours or intravenous methylprednisolone $3 \mathrm{mg} / \mathrm{kg} /$ day) were given in a tailing dose for 21 days if there was no clinical or radiological response with antibiotics and the clinical criteria for SARS were fulfilled. The introduction of ribavirin and steroids was hastened if there was a definite history of close contact with known SARS patients such as occupational or 
Table 1 Analysis of factors affecting mortality using the univariate Cox proportional hazards model

\begin{tabular}{|c|c|c|}
\hline & Hazards ratio $(95 \% \mathrm{Cl})$ & $p$ value \\
\hline Sex (male) & $1.6(0.6$ to 4.0$)$ & 0.320 \\
\hline Age (>60 years) & $6.8(2.5$ to 18.4$)$ & $<0.001$ * \\
\hline Presence of comorbidity & 9.0 (3.2 to 25.3$)$ & $<0.001$ * \\
\hline Diabetes mellitus (DM) & $4.7(1.5$ to 14.3$)$ & $0.008 *$ \\
\hline Cardiac disease & $9.2(3.5$ to 23.9$)$ & $<0.001$ * \\
\hline Presence of DM and/or cardiac disease $†$ & 12.5 (4.1 to 37.7$)$ & $<0.001^{*}$ \\
\hline Presence of other comorbidities $\dagger$ & $5.6(1.5$ to 20.8$)$ & $0.010 *$ \\
\hline Multilobar radiological abnormality & $2.0(0.8$ to 5.0$)$ & 0.167 \\
\hline Temperature $\left({ }^{\circ} \mathrm{C}\right) \ddagger$ & $1.4(0.7$ to 3.0$)$ & 0.335 \\
\hline Oxygen saturation $(\%) \ddagger$ & $0.9(0.8$ to 1.1$)$ & 0.399 \\
\hline Total white cell count $\left(10^{\circ} / 1\right) \ddagger$ & $1.1(1.0$ to 1.2$)$ & 0.153 \\
\hline Neutrophil $\left(10^{9} / 1\right) \ddagger$ & $1.1(1.0$ to 1.2$)$ & 0.073 \\
\hline Lymphocyte $\left(10^{9} / \mathrm{I}\right)$ & $2.3(0.6$ to 8.0$)$ & 0.205 \\
\hline Platelet count $\left(10^{\circ} / /\right) \ddagger$ & $1.0(1.0$ to 1.0$)$ & 0.823 \\
\hline Serum sodium $(\mathrm{mmol} / \mathrm{l}) \ddagger$ & $1.0(0.9$ to 1.2$)$ & 0.857 \\
\hline Serum urea $(\mathrm{mmol} / \mathrm{l}) \ddagger$ & $1.2(1.0$ to 1.4$)$ & $0.017^{*}$ \\
\hline Alanine aminotransferase (IU/I) $\ddagger$ & $1.0(1.0$ to 1.0$)$ & 0.097 \\
\hline Prolonged INR (>1.2 seconds) & $3.1 \quad(0.7$ to 13.4$)$ & 0.135 \\
\hline Prolonged aPTT (>42 seconds) & $3.3(1.2$ to 8.9$)$ & $0.018^{*}$ \\
\hline High creatine kinase (>330 IU/I) & $2.3(0.9$ to 6.1$)$ & 0.100 \\
\hline High lactate dehydrogenase (>450 IU/I) & $4.8(1.4$ to 16.6$)$ & $0.014^{*}$ \\
\hline
\end{tabular}

household contacts. The protocol using methylprednisolone and the theoretical background of using this combination treatment has recently been described in detail. ${ }^{10}$ Pulse methylprednisolone, in a daily dose of $500 \mathrm{mg}$ to $1 \mathrm{~g}$ for 2 days, was given if the clinical condition, radiological presentation, or oxygen saturation worsened (at least two of the three) and lymphopenia persisted. A standard corticosteroid regimen was continued after the pulse steroid therapy. A concomitant switch to more potent antibiotics with increased coverage of Gram negative organisms was also made when pulse steroids were considered necessary. Patients who required mechanical ventilation or those who could not be maintained at an oxygen saturation level of $>90 \%$ despite oxygen supplementation of $\geqslant 4 \mathrm{l} / \mathrm{min}$ were transferred to the ICU for close monitoring.

The clinical, demographical, laboratory and radiological findings of the patients were collected and analysed. The primary end point was death, and the secondary end point (adverse clinical outcome) was defined as the need for mechanical ventilation or death.

\section{Data analysis}

Data were reported as mean (SD) unless specified otherwise. The time to death and time to an adverse outcome were investigated using survival analysis with follow up starting at hospital admission and ending on 31 May 2003. Adverse outcome was defined as the need for mechanical ventilation or death. Patients were censored if at the end of follow up they were still alive (for mortality) or did not have an adverse outcome (for adverse outcome). The Kaplan-Meier method was used for time-to-event plot. Comparisons between groups of time-toevent data were made using the Cox proportional hazards model. A multivariate Cox proportional hazards model was used to look for independent risk factors for mortality from the three variables: presence of diabetes mellitus or cardiac disease, presence of other comorbid conditions, or age above 60. A similar method was used to identify risk factors for adverse outcomes. SPSS (version 11.0) was used for all statistical analyses.

\section{RESULTS}

One hundred and fifteen patients were admitted to Queen Elizabeth Hospital between 9 March and 31 May 2003. Eighty two patients were discharged and 18 died, giving a crude mortality rate of $15.7 \%$ and a 21 day mortality rate of 10 (SE 3)\%. Thirty nine patients (34\%) required ICU admission and 30 patients $(26 \%)$ required mechanical ventilation. The median follow up time was 62 days (interquartile range (IQR) 47.7573.0). The mean (SD) age of the patients was 41.0 (14.8) years. There were 45 (39\%) men and 106 (92\%) were Chinese. Twenty six patients $(22.6 \%)$ had one or more comorbidities (diabetes mellitus, hypertension, ischaemic heart disease, congestive heart failure, asthma, cerebrovascular accident, and chronic renal failure). Fifteen (13\%) had travelled to mainland China within 2 weeks of the onset of symptoms and $24(21 \%)$ were healthcare workers (five doctors, 18 nurses, and one paramedic).

The presenting symptoms included fever $(100 \%)$, chills (55\%), cough $(46 \%)$, myalgia (38\%), malaise $(35 \%)$, sputum production $(15 \%)$, headache (11\%), diarrhoea (11\%), sore throat $(7 \%)$, and dyspnoea (10\%). The mean (SD) time between onset of symptoms and admission was 3.6 (2.4) days. Mean (SD) temperature upon presentation was $38.9(0.6)^{\circ} \mathrm{C}$, oxygen saturation 96.9 (2.5)\%, systolic blood pressure 120.8 (17.1) $\mathrm{mm} \mathrm{Hg}$, and pulse rate 91.7 (17.3) beats/min. Common presenting laboratory findings included lymphopenia (mean (SD) lymphocyte count $\left.0.87(0.37) \times 10^{9} / \mathrm{l}\right)$ and raised lactate dehydrogenase (LDH) (mean (SD) 562.5 (351.4) IU/1). Raised serum alanine aminotransferase (ALT) (mean (SD) 35.83 (26.5) U/l) and severe thrombocytopenia (mean initial platelet count $\left.184.3(73.3) \times 10^{9} / 1\right)$ were not commonly found on presentation in our cohort. Initial chest radiographs were clear in 15 patients $(13 \%)$ where high resolution CT scans of the thorax were used to detect the presence of pneumonia. 26 patients $(22.6 \%)$ presented with multilobar lung abnormalities.

The RT-PCR test for coronavirus was only available at the end of March. The overall positivity rate in our cohort was $41 \%$, with a higher sensitivity in stool samples (20/28, sensitivity $71.4 \%$ ) than in respiratory samples (nasopharyngeal aspirates $(18 / 50,36 \%)$, tracheal aspirates $(6 / 20,30 \%)$, or throat swabs $(28 / 92,30.4 \%))$. The seroconversion rate for coronavirus IgG antibodies at the time of writing was $89 \%$.

With the exception of two patients who developed acute myocardial infarction during their hospital stays, all the 


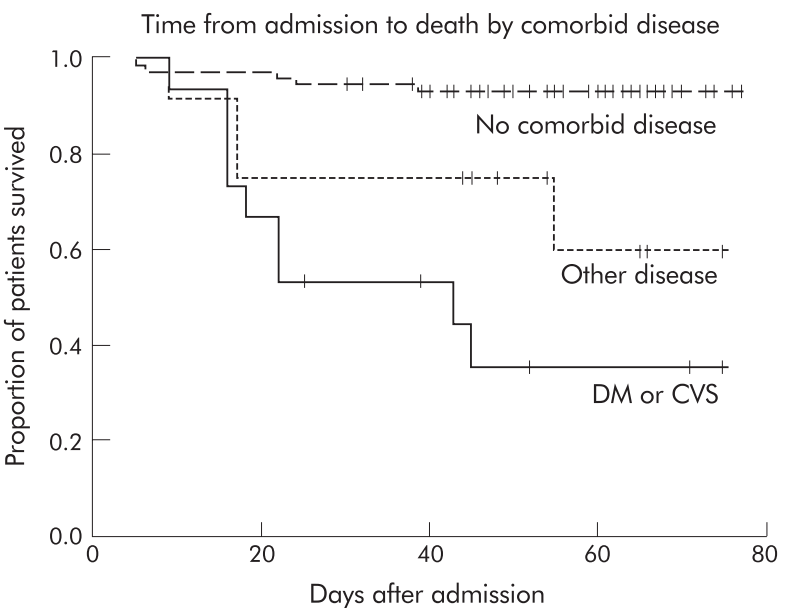

Figure 1 Survival analysis showing time from admission to death by comorbid conditions. DM=diabetes mellitus; $\mathrm{CVS}=$ cardiovascular disease.

patients died of respiratory failure as a result of acute respiratory distress syndrome due to SARS.

\section{Factors affecting mortality rate}

The univariate Cox proportional hazards model showed that the mortality risk was 6.8 times higher in those aged above 60 $(95 \%$ CI 2.5 to $18.3 ; p<0.001)$. For every 10 year increase in age the risk of death increased by 1.9. The presence of comorbidities increased the mortality risk (HR 9.0, 95\% CI 3.2 to 25.3; $\mathrm{p}<0.001$ ), with cardiac disease (HR 9.2, 95\% CI 3.5 to 23.9; $\mathrm{p}<$ 0.001 ) and diabetes mellitus (HR 4.7, 95\% CI 1.5 to 14.3; $\mathrm{p}=0.008$ ) being the most important comorbidities (table 1 ). The presence of diabetes and/or cardiac disease was associated with an increased mortality risk of 12.5 (95\% CI 4.1 to 37.7; $\mathrm{p}<0.001$ ), while the presence of other comorbid conditions was associated with an increased mortality risk of 5.6 (95\% CI 1.5 to $20.8 ; \mathrm{p}<0.001$, fig 1 ). Other factors that significantly affected mortality included prolonged thromboplastin time, increased urea level, and raised lactate dehydrogenase levels (>450 IU/l; table 1).

The multivariate Cox proportional hazard model was used to look for independent risk factors for mortality. Using a model with age above 60, presence of diabetes and/or cardiac diseases, and the presence of other comorbidities, it was found that the presence of diabetes mellitus and/or cardiovascular disease (HR 9.1, 95\% CI 2.8 to 29.1, p $<0.001$ ), the presence of other comorbidities (HR 5.2, 95\% CI 1.4 to $19.7 ; p=0.01$ ), and age above 60 (HR 3.5, 95\% CI 1.2 to $10.2 ; \mathrm{p}=0.03$ ) were all independent predictors of death (table 2).

\section{Factors affecting adverse outcomes}

In the analysis of risk factors affecting adverse outcomes using the univariate Cox proportional hazards model, it was found that age above 60 (HR 2.6, 95\% CI 1.0 to 6.9; $\mathrm{p}=0.048$ ), the presence of any comorbidities (HR 5.0, 95\% CI 2.4 to 10.3; $\mathrm{p}<0.001)$, and multilobar involvement on the chest radiograph (HR 2.6, 95\% CI 1.2 to 5.4; $p=0.011$ ) were significant. As with mortality, the presence of diabetes mellitus and/or cardiac disease was found to be significant in affecting adverse outcomes (HR 8.2, 95\% CI 3.7 to 18.2 , p <0.001), but the presence of other comorbid conditions as a group was not statistically significant $(\mathrm{p}=0.065)$.

The multivariate Cox proportional hazards model was used to look for independent risk factors for adverse outcomes. In a model with age above 60, presence of multilobar radiograph involvement, presence of diabetes or cardiac disease, and the presence of other comorbidities, only the presence of diabetes mellitus or cardiac disease was found to be an independent risk factor for an adverse outcome (HR 7.3, 95\% CI 3.1 to 17.4, $\mathrm{p}<0.001$ ).

\section{DISCUSSION}

This study aimed to determine the short term outcome and risk factors on presentation that were associated with an adverse clinical course and mortality in a group of predominantly Chinese patients with SARS. Age above 60 and the presence of comorbid conditions were associated with a higher rate of mortality. The presence of diabetes mellitus and/or cardiac diseases was related both to a higher mortality rate and an adverse outcome. The crude mortality rate of $15.7 \%$ and 21 day mortality rate of $10 \%$ (standard error $3 \%$ ) appeared higher than in other recently published studies ${ }^{5-7}$ but, as pointed out by Donnelly et al, ${ }^{11}$ it is difficult to calculate the true mortality rate of the disease while the epidemic is still continuing and it is impossible to ascertain which of the remaining patients will eventually die or be discharged. The estimated case fatality rate calculated by Donnelly et al was $13.2 \%$ for patients younger than 60 and more than $40 \%$ for those aged over $60 .{ }^{11}$

The presence of comorbidities, especially diabetes mellitus and cardiac diseases, was found to have an important effect on the outcome of our patients. The effects of age $\mathrm{ag}^{5-7}$ and comorbidities ${ }^{6}$ have also been addressed in other cohorts of patients with SARS. This is not surprising as these have been shown to increase the risk of death or a complicated course in patients with community acquired pneumonia (CAP). ${ }^{12}$ Diabetes mellitus has also been identified as a prognostic factor for death in patients with CAP. ${ }^{13}$ The presence of diabetes mellitus could predispose to superimposed nosocomial pneumonia due to Staphylococcus aureus ${ }^{14}$ or other agents in these already critically ill patients, although this was not observed in our patients. The presence of a suboptimal cardiac status together with a fulminant chest condition could lead to compromised haemodynamic status, especially when concomitant superinfections and anaemia are present.

Apart from the inherently severe nature of the infection caused by this novel virus, the potentially serious side effects of the treatment agents might also contribute to the observed mortality and morbidity associated with SARS in this group of relatively young patients. All of our patients received ribavirin and steroids in accordance with the guidelines of the local

Table 2 Multivariate analysis showing the independent risk factors for mortality using the Cox proportional hazards model

\begin{tabular}{lll}
\hline & Hazards ratio $(95 \% \mathrm{Cl})$ & p value \\
\hline Age $>60$ years & $3.5(1.2$ to 10.2$)$ & $0.02^{*}$ \\
Presence of DM/cardiac disease & $9.1(2.8$ to 29.1$)$ & $<0.001^{*}$ \\
Presence of other comorbidities $\dagger$ & $5.2(1.4$ to 19.7$)$ & $0.01^{*}$ \\
\hline
\end{tabular}

DM=diabetes mellitus

${ }^{*} \mathrm{p}<0.05$.

tOther comorbidities include hypertension, chronic lung diseases, cerebrovascular accidents.

Reference group is patient under 60 with no history of DM or cardiac disease and no history of other comorbidities. 
hospital authority. Similar treatment protocols have also been used in other hospitals in Hong Kong and Canada, ${ }^{6710}$ but there has been some controversy with the use of these agents in the treatment of this condition. ${ }^{15}{ }^{16}$ The use of high dose corticosteroids early in the course of an infective disease is controversial. In a small randomised study corticosteroids were found to be useful only in the late phase of acute respiratory distress syndrome (ARDS), ${ }^{17}$ but its use in ARDS is not well supported and more evidence from larger prospective studies is needed. The dose of steroids used in Canada was also much lower than that used in Hong Kong. ${ }^{6}$ However, corticosteroids have also been found not to be useful in lower respiratory infections caused by respiratory syncytial virus. ${ }^{18}$ Conditions observed in our patients that might have been at least indirectly related to the use of high dose corticosteroids included hyperglycaemia in non-diabetic patients (15\%), hypokalaemia (39\%), gastrointestinal bleeding (3\%), and hypertension $(7 \%)$. Whether corticosteroids would be more useful in the later phases of the disease process when the immunological processes are activated with cytokines is debatable. Although no haemolysis was observed, anaemia was found in $34 \%$ of our patients during the course of the disease that could at least be partially caused by ribavirin, an agent with numerous side effects. ${ }^{19}$ Unfortunately, in the absence of sufficient in vitro data and randomised controlled studies it is not possible to draw any firm conclusion on the efficacy of the existing regimen at this time.

The rate of admission to the ICU in our patients was high, with $76.9 \%$ requiring mechanical ventilation. The high rate of ICU admissions might be related to our relatively early initiation of close monitoring of the patients. If intubation was considered necessary it was performed in a controlled manner in the ICU because there have been suspected reports of contracting the infection during resuscitations, emergency intubation, and manual bagging of patients. Controlled elective intubation in the ICU enables healthcare workers to be better prepared and protected than during emergency procedures carried out in an ordinary isolation ward.

No laboratory parameter was identified as an independent predictor of adverse outcomes, including raised $\mathrm{LDH}$ and a high neutrophil count described by Lee et al. ${ }^{5}$ Although lymphopenia has been commonly observed, the absolute lymphocyte count was not associated with adverse outcomes in our study.

The diagnosis of SARS is still clinical at present, based on a number of parameters that might encompass other infections. Although the novel coronavirus has been described, the reliability of the RT-PCR test for the virus needs to be improved. The overall sensitivity of the RT-PCR test for coronavirus in our patients was only $41 \%$. This might be affected by the source of the samples, the technique of obtaining the samples, as well as the viral load of the patient when the samples were obtained. The timing of seroconversion might also be affected by the use of high dose steroids in these patients. When all the serological results of the coronavirus become available for all these patients, it might be able to focus on the entities "coronavirus related SARS" or "coronavirus pneumonia" and the subsequent analyses of clinical data of these patients would be more meaningful and reliable. At this stage it seems that the short term outcomes can vary quite significantly between centres, hence the need for larger multicentre studies to give a more reliable picture of this new disease.

\section{Authors' affiliations}

J W M Chan, C K Ng, Y H Chan, W H O, S Lee, S Y Y Chu,

W L Law, M P Lee, P C K Li, Department of Medicine, Queen Elizabeth Hospital, Hong Kong SAR, China

T Y W Mok, Respiratory Medical Department, Kowloon Hospital, Hong Kong SAR, China

\section{REFERENCES}

1 Ksiazek TG, Erdman D, Goldsmith CS, et al. A novel coronavirus associated with severe acute respiratory syndrome. N Engl J Med 2003:348:1953-66.

2 Drosten C, Gunther S, Preiser W, et al. Identification of a novel virus in patients with severe acute respiratory syndrome. N Engl J Med 2003;348: 1967-76.

3 Peiris JSM, Lai ST, Poon LLM, et al. Coronavirus as a possible cause of severe acute respiratory syndrome. Lancet 2003:361:1319-25.

4 Tsang KW, Ho PL, Ooi GC, et al. A cluster of cases of severe acute respiratory syndrome in Hong Kong. N Engl J Med 2003;348:1977-85.

5 Lee N, Hui D, Wu A, et al. A major severe outbreak of severe acute respiratory syndrome in Hong Kong. N Engl J Med 2003;348:1986-94.

6 Booth CM, Matuka LM, Tomlinson GA, et al. Clinical features and short-term outcomes of 144 patients with SARS in the Greater Toronto area. JAMA 2003;289:2801-9.

7 Peiris JSM, Chu CM, Cheng VCC, et al. Clinical progression and viral load in a community outbreak of coronavirus-associated SARS pneumonia: a prospective study. Lancet 2003;361:1767-72.

8 Hospital Authority of Hong Kong. HA guidelines on severe acute respiratory syndrome. Available at http://www.ha.org.hk/sars/ guidelines/index.html (accessed 10 May 2003).

9 World Health Organisation. Case definitions for surveillance of severe acute respiratory syndrome (SARS). Available at http://who.int/csr/ sars/casedefinition/en (accessed 10 May 2003).

10 So LKY, Lau ACW, Yam LYC, et al. Development of a standard treatment protocol for severe acute respiratory syndrome. Lancet 2003;361:1615-7.

11 Donnelly CA, Ghani AC, Leung GM, et al. Epidemiological determinants of spread of casual agent of severe acute respiratory syndrome in Hong Kong. Lancet 2003;361:1761-6.

12 Niederman MS, Mandell LA, Anzueto A, et al. American Thoracic Society guidelines for the management of adults with community-acquired pneumonia. Am J Respir Crit Care Med 2001;163:1730-54.

13 Fine MJ, Smith MA, Carson CA, et al. Prognosis and outcomes of patients with community-acquired pneumonia: a meta-analysis. JAMA 1996;275: 134-41.

14 Rello J, Quintana E, Ausina V, et al. Risk factors for Staphylococcus aureus nosocomial pneumonia in critically ill patients. Am Rev Respir Dis 1990; 142:1320-4

15 Centres of Disease Control and Prevention. Severe acute respiratory syndrome and coronavirus testing-United States, 2003. http://www.cdc.gov/mmwr/preview/mmwrhtml/mm5214al.htm. laccessed 10 May 2003).

16 Oba Y, Lee N, Sung J. The use of corticosteroids in SARS. N Engl J Med 2003;348:2034-5

17 Meduri GU, Headley AS, Golden E, et al. Effect of prolonged methylprednisolone therapy in unresolving acute respiratory distress syndrome: a randomized controlled trial. JAMA 1998;280:159-65.

18 Van Woensel JBM, Van Aalderen WMC, de Weerd W, et al. Dexamethasone for treatment of patients mechanically ventilated for lower respiratory tract infection caused by respiratory syncytial virus. Thorax 2003;58:383-7

19 Koren G, King S, Knowles S, et al. Ribavirin in the treatment of SARS: a new trick for an old drug? Can Med Assoc J 2003;168: 1289-92. 